November 15, 2017

\title{
INSTANTON-INDUCED CORRELATIONS IN HADRONS
}

\author{
PIETRO FACCIOLI* \\ E.C.T.* 286 Strada delle Tabarelle, Villazzano (Trento) Italy, I-38050 and \\ INFN, Gruppo Collegato di Trento.
}

\begin{abstract}
QCD instantons generate non-perturbative spin- and flavor- dependent forces between quarks. We review the results of a series of studies on the role played by instanton-induced correlations in hadrons. We first present a study of instanton-mediated interactions in QCD, based on lattice simulations. Then we show that the Instanton Liquid Model can reproduce the available data on proton and pion form factors at large momentum transfer. The virial expansion in the vacuum diluteness parameter can explain why the perturbative regime sets in very early in some physical processes and much later in some other. We discuss the connection with diquarks. Instantons generate a deeply bound scalar color anti-triplet diquark, with a mass of about $450 \mathrm{MeV}$ and electric charge radius $\sim 0.7 \mathrm{fm}$. The strong attraction in the $\overline{\mathbf{3}}_{\mathbf{c}} 0^{+}$diquark channel leads to a quantitative description of non-leptonic decays of hyperons and provides a microscopic dynamical explanation of the $\Delta I=1 / 2$ rule.
\end{abstract}

Keywords: Instantons; lattice QCD; diquarks; form factors; weak decays.

\section{Non-perturbative correlations in hadrons}

Several recent experiments have shown that strong non-perturbative forces inside hadrons survive also at relatively large momentum transfer, $Q^{2} \gtrsim 2-6 \mathrm{GeV}^{2}$. Evidence for this fact has come, for example, from the measurement of the pion space-like form factor up to $Q^{2} \simeq 2 \mathrm{GeV}^{2}$ [1] and from the direct determination of the proton $G_{E}\left(Q^{2}\right) / G_{M}\left(Q^{2}\right)$ ratio up to $Q^{2} \simeq 6 \mathrm{GeV}^{2}$ [2]. In these reactions, the data are very far from the asymptotic perturbative QCD prediction. This behavior strongly contrasts with the observed perturbative scaling of the DIS structure functions, for $Q^{2} \gtrsim 1 \mathrm{GeV}^{2}$.

It is tempting to argue that the delay in the onset of the perturbative regime in the electro-magnetic form factors simply reflects the fact that exclusive processes are very sensitive to the non-perturbative dynamics of hadronization. However, this explanation is ruled-out by experiment: in fact, we know of at least one exclusive observable, the $\gamma \gamma^{*} \rightarrow \pi_{0}$ transition form factor, which gently follows the perturbative prediction, already for $Q^{2} \gtrsim 1-2 \mathrm{GeV}^{2}$.

The JLAB results on form factors have provided new important insight on the interactions inside hadrons. The evidence for non-perturbative correlations at rather short distances, of the order of the tenth of the fermi, rules-out models in which

*email: faccioli@ect.it 
the quarks move essentially as free particle inside a confining bag and feel the nonperturbative color field only when they approach the edge of the hadron, i.e. for distances of the order $\sim 1 \mathrm{fm}$.

From the comparison of the pion space-like form factor (which deviates from the perturbative prediction) and the $\gamma \gamma^{*} \rightarrow \pi_{0}$ transition form factor (which follows the perturbative prediction) we have to conclude that the non-perturbative short-range interactions are strongly channel dependent: they are effective in some process, but they are almost absent in some other. This property of the non-perturbative dynamics has been known for a long time: another example is the "Zweig rule" forbidding flavor-mixing, which works very well in the vector and axial vector meson channels, but it is violated in the scalar and pseudo-scalar channels.

The channel-dependence of the non-perturbative correlations implies that the quark-quark interaction at low-energy has much more structure than a simple radial potential. It must at least be spin-dependent. Moreover, the rather large flavor asymmetry observed in DIS parton distributions suggests that it is also flavordependent. As a consequence, quarks are more correlated in some spin and flavor configuration than in other. Indeed, a number of phenomenological studies seem to indicate that quarks preferably correlate to form color- and flavor- anti-triplet scalar diquarks (see [3] and references therein).

An important testing ground for the spin-flavor structure of the non-perturbative quark-quark interaction is represented by weak-decays of hadrons. The natural scale of weak processes - set by $W$ boson mass - is much larger than all other scales involved in the hadron internal dynamics. Hence, weak interactions can be regarded as effectively local and can resolve small structures inside hadrons. Moreover, their explicit dependence on flavor and chirality can be exploited to probe the spin and flavor structure of the non-perturbative QCD interaction. Among the large variety of weak hadronic processes, a prominent role is played by the non-leptonic decays of kaons and hyperons, which are characterized by the famous $\Delta I=1 / 2$ rule. Neither electro-weak nor perturbative QCD interactions can account for the dramatic relative enhancement of the $\Delta I=1 / 2$ decay channels. Its origin must therefore reside in the non-perturbative sector of QCD. Stech, Neubert and collaborators observed that the experimental data on both hyperon and kaon decays could be understood by assuming strong diquark correlations in the scalar, color anti-triplet channel [4].

The evidence for short-range, spin-dependent non-perturbative correlations in hadrons naturally leads to the problem of identifying their dynamical origin. To this end, it is instructive to analyze the non-perturbative scales in QCD. We know of at least two non-perturbative phenomena which occur at a momentum scale higher than $\Lambda_{Q C D}$ : the dynamical breaking of chiral symmetry and the anomalous breaking of the axial symmetry. The natural scale for the interactions related to chiral symmetry breaking is set by the mass of the lightest vacuum excitation which is not protected by chiral symmetry, the $\rho$-meson. Similarly, the typical scale of topological interactions is given by the mass of the $\eta^{\prime}$ meson. Hence, we should not be surprised to find non-perturbative effects at the $\mathrm{GeV}$ scale. 
The physical properties of the pion are certainly strongly influenced by the interactions responsible for the breaking of chiral symmetry. Presumably, these forces play an important role in the lightest baryons.

\section{Why instantons?}

Instantons have been proposed long ago as the dynamical mechanism driving both the saturation of the axial anomaly [5] and the spontaneous breaking of chiral symmetry [6] [7] ( for a review see [8] ). The this hypothesis has been checked in a number of lattice-based studies 9 .

Physically, instantons are gluon fields which are generated during tunneling events between degenerate QCD vacua. Mathematically, they are non-perturbative solutions of the Euclidean Yang-Mills equation of motion. Being minima of the YangMills action, they have been used in the context of a saddle-point (semi-classical) analysis of the Euclidean QCD path integral [5].

In the Instanton Liquid Model (ILM) the functional integral over all possible gluon field configurations is replaced by a sum over the configurations of an ensemble of instantons and anti-instantons. The path integral can then be solved by exploiting the formal analogy between the Euclidean QCD generating functional and the partition function of a grand-canonical statistical ensemble. The phenomenological parameters in the model are the average instanton density $\bar{n} \simeq 1 \mathrm{fm}^{-4}$ - which relates to the rate of tunneling in the vacuum - and the average instanton size $\bar{\rho} \simeq 1 / 3 \mathrm{fm}-$ which determines how long each tunneling event lasts for - . These values were extracted more than two decades ago from the global vacuum properties [6] and indicate that the diluteness (or "packing fraction") of the instanton ensemble is a small parameter: $\kappa=\bar{n} \bar{\rho}^{4} \simeq 0.01$. Tunnelings are fairly rare events.

The instanton field induces an effective vertex between quarks ('t Hooft interaction) which, for $Q^{2} \ll 1 / \bar{\rho}^{2}$, reduces to a $2 N_{f}$-leg contact interaction. For example, for $N_{f}=2$ it reads:

$$
\mathcal{L}^{\prime} t H=G_{\bar{\rho}, \bar{n}}\left(\left[\left(\psi^{\dagger} \tau_{a}^{-} \psi\right)^{2}-\left(\psi^{\dagger} i \gamma_{5} \tau^{-} \psi\right)^{2}\right]+\frac{1}{2\left(2 N_{c}-1\right)}\left(\psi^{\dagger} \sigma_{\mu \nu} \tau_{a}^{-} \psi\right)^{2}\right),
$$

where $\tau^{-}=(\vec{\tau}, i)\left(\vec{\tau}\right.$ are isospin Pauli matrices), and $G_{\bar{n} \bar{\rho}}$ is a coupling constant depending on the typical density and size of instantons in the vacuum. The finite size of the instanton field provides a natural cut-off scale for the 't Hooft interaction.

The non-perturbative instanton-induced interaction (1) has two important characteristic features: (i) it involves quarks of different flavor, (ii) it is chirality-mixing, i.e. quarks must flip their chirality any time they cross the field of an instanton. These two properties distinguish the instanton-induced interaction from the perturbative quark-gluon vertex, which is flavor-blind and chirality-conserving.

The chirality-flipping nature of the 't Hooft vertex allows to understand why instanton effects are strongly channel dependent. In fact, processes in which a quark 
undergoes a chirality-flip can occur any time there is an instanton nearby. In this case, the corresponding matrix elements will get a contribution from the instantoninduced interaction at the leading order in the instanton packing fraction, i.e. $o(\kappa)$. Conversely, transitions in which quarks do not change their chirality get contribution from instantons only at the next-to-leading order in the packing fraction, i.e. $o\left(\kappa^{2}\right)$. The physical reason is that the probability for two tunneling events to occur during the same scattering process is suppressed, if tunnelings are sufficiently rare events.

This mechanism explains the suppression of non-perturbative effects in several channels. A good example is the suppression of flavor-mixing in the vector and axialvector meson channels (Zweig rule). The Lagrangian (1) induces flavor-mixing. Due to its chirality-flipping structure, its contribution to the scalar and pseudo-scalar channels is of $o(\kappa)$, so maximally enhanced. On the other hand, its contribution to the vector and axial-vector channels is of $o\left(\kappa^{2}\right)$, so much smaller.

The chirality-flipping structure of the 't Hooft Lagrangian (1) has been used to look for signatures of instanton-induced interaction by means of lattice QCD simulations [10. If the interaction between light quarks is mainly instanton-mediated, then we expect a new chirality-flip any time quarks cross the field of an instanton. Based on this observation, one is led to consider the "chirality-flip" asymmetry constructed by taking the ratio of the amplitude for a $|u \bar{d}\rangle$ pair to be found after an interval $\tau$ in a state in which the chiralities of the quark and the anti-quark are interchanged, relative to the amplitude to remain in the same chirality state [11. It can be shown that, in the quantum field theory formalism, such a ratio reads:

$$
R(\tau)=\frac{A_{\text {flip }}(\tau)}{A_{\text {non-flip }}(\tau)}=\frac{\Pi_{P S}(\tau)-\Pi_{S}(\tau)}{\Pi_{P S}(\tau)+\Pi_{S}(\tau)},
$$

where

$$
\begin{aligned}
\Pi_{P S}(\tau) & =\left\langle 0\left|J_{P S}(\tau) J_{P S}^{\dagger}(0)\right| 0\right\rangle, \quad J_{P S}(x)=\bar{u}(x) i \gamma_{5} d(x), \\
\Pi_{S}(\tau) & =\left\langle 0\left|J_{S}(\tau) J_{S}^{\dagger}(0)\right| 0\right\rangle, \quad J_{S}(x)=\bar{u}(x) d(x) .
\end{aligned}
$$

Notice that the ratio $R(\tau)$ must vanish as $\tau \rightarrow 0$ (no chirality flips), and must approach 1 as $\tau \rightarrow \infty$ (infinitely many chirality flips).

The instanton picture gives a very specific prediction for the asymmetry (2). If quarks propagate in the vacuum for a time comparable with the typical interval between two consecutive tunneling events, they have a large probability of crossing the field of an instanton. Hence, after some time, the quarks are most likely to be found in the configuration in which their chirality is flipped and $R(\tau)>1$. However, if one waits longer, then the quarks will "bump" into another instanton field, which will re-flip their chirality and restore the initial chirality state. So, the probability to find the quark and antiquark in the flipped chirality state will start decreasing. Hence, the instanton picture predicts that the function $R(\tau)$ will have a maximum at $\tau \sim \bar{n}^{1 / 4}$.

Such a characteristic prediction of the instanton model has been checked on the lattice. In Fig. 1 (left panel) we compare the results of a quenched ILM simulation 

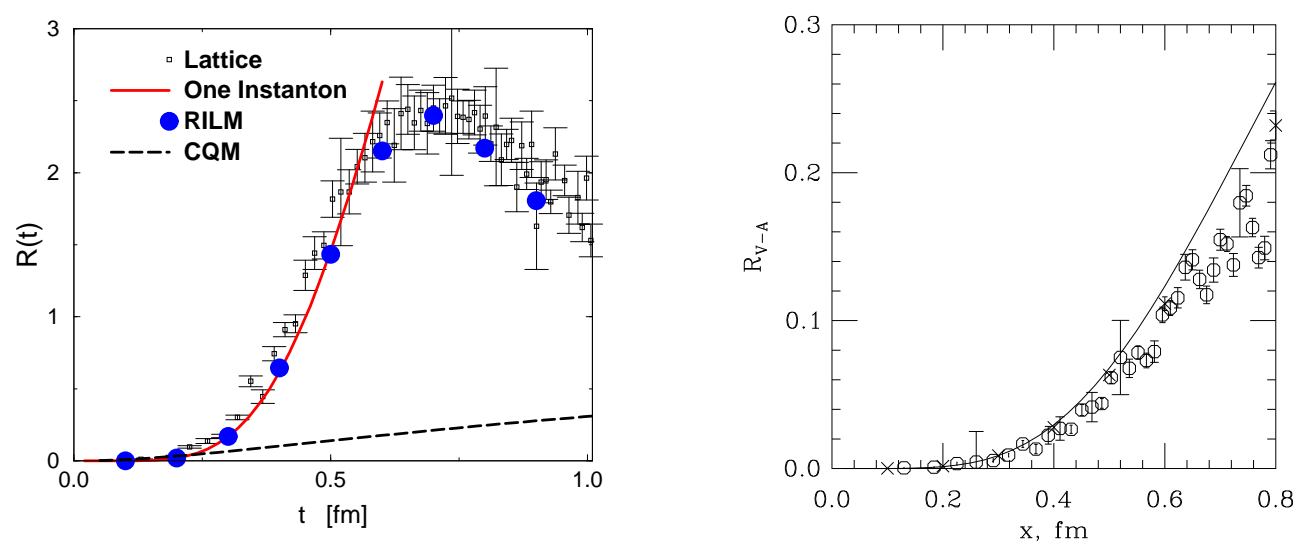

Fig. 1.

LEFT PANEL: The chirality-flip correlator (2) in lattice QCD (square points) and in the ILM (circles). The solid line represent the single-instanton contribution. The dashed curve was obtained from two free "constituent" quarks with a mass of $400 \mathrm{MeV}$.

RIGHT PANEL: The ratio (5), evaluated from lattice QCD (octagons) -extrapolated to zero quark mass-, from the ILM (crosses) and extracted from the ALEPH $\tau$-lepton decay data, as extracted in Ref. 13 (lines).

with the result of a quenched lattice calculation, performed using the same bare quark masses and the same four-dimensional box. The agreement between the ILM and QCD is impressive, even at the quantitative level (note that no-parameter fitting was involved in the ILM calculation).

A similar quantitative agreement between ILM and lattice simulations has been observed by DeGrand also in the asymmetry constructed with vector and axialvector two-point correlation functions [12]:

$$
R(\tau)=\frac{\Pi_{V}(\tau)-\Pi_{A V}(\tau)}{\Pi_{V}(\tau)+\Pi_{A V}(\tau)}
$$

where

$$
\begin{aligned}
\Pi_{V}(\tau) & =\left\langle 0\left|J_{\mu}(\tau) J_{\mu}^{\dagger}(0)\right| 0\right\rangle, \quad J_{\mu}(x)=\bar{u}(x) \gamma_{\mu} d(x), \\
\Pi_{A V}(\tau) & =\left\langle 0\left|J_{\gamma_{\mu 5}}(\tau) J_{\gamma_{\mu 5}}^{\dagger}(0)\right| 0\right\rangle, \quad J_{\gamma_{\mu 5}}(x)=\bar{u}(x) \gamma_{\mu} \gamma_{5} d(x) .
\end{aligned}
$$

The results are presented in Fig. 1 (right panel). We recall that the vector and axial-vector point-to-point correlators receive contribution from instantons only at $o\left(\kappa^{2}\right)$. It is quite remarkable that the instanton-induced effects provide the correct amount of non-perturbative correlations even when they are relatively suppressed: there seems to be little room for additional non-perturbative dynamics, in these correlators.

This collection of lattice results represents a compelling evidence for large instanton contributions to the dynamics of light quark. On the other hand, it should be mentioned that instantons couple much more weakly to heavy quarks, and that the discussion of their role in pure gluon-dynamics is still rather controversial. 

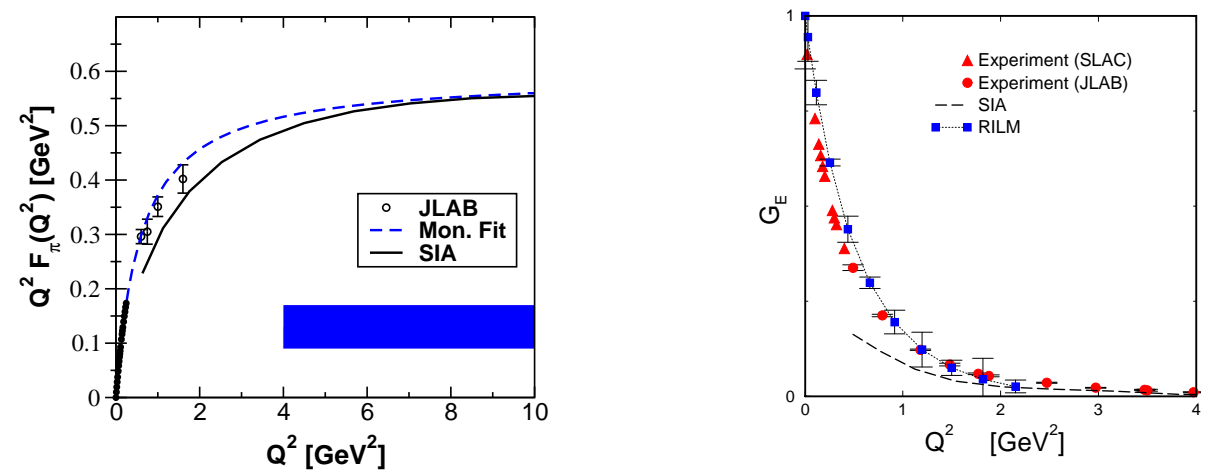

Fig. 2.

LEFT PANEL: The JLAB data for $Q^{2} F_{\pi}\left(Q^{2}\right)$ in comparison with the asymptotic perturbative QCD prediction (thick bar, for a typical $\alpha_{s} \sim 0.2-0.4$ ), the monopole fit (dashed line), and the SIA calculation (solid line). The SIA calculation is not reliable below $Q^{2} \sim 1 \mathrm{GeV}^{2}$. The solid circles denote the SLAC data.

RIGHT PANEL: The electric form factor of the proton in the ILM and from experiment. Triangles are low-energy SLAC data, which follow a dipole fit. Circles are experimental data obtained from the recent JLAB result for $G_{E} / G_{M}$, assuming a dipole fit for the magnetic form factor. Squares are result of many-instanton simulations in the ILM, and the dashed line is the SIA curve.

From this discussion it follows that instantons represent natural candidates for the microscopic dynamical mechanism underlying the short-range non-perturbative correlations in light hadrons. In the following sections we shall show that the ILM quantitatively reproduces the JLAB data on electro-magnetic form factors and explains why the perturbative regime sets-in very early in DIS and in the $\gamma^{*} \gamma \rightarrow \pi_{0}$ transition form factor. Moreover, we will also show that instantons generate a scalar, color anti-triplet diquark bound-state of roughly $450 \mathrm{MeV}$ mass. Such strong instanton-induced diquark correlations lead to a quantitative understanding of nonleptonic weak decays of hyperons and to an explanation of the $\Delta I=1 / 2$ rule.

\section{Instantons and the electro-magnetic structure of hadrons}

Let us first discuss the instanton contribution to the electro-magnetic form factors. The framework to compute momentum-dependent hadronic matrix elements, from vanishing to large momentum transfer, has been recently developed in a number of papers [15] 16] 17] 19]. At large momentum transfer the calculations of hadronic matrix elements in the ILM can even be carried-out analytically, by means of the Single Instanton Approximation (SIA) 20. This approach exploits the fact that small-sized correlation functions are dominated by the interaction of the quarks with a single instanton. At small or vanishing momentum transfer, many-instanton effects are important and one has to rely on numerical Monte Carlo methods to compute the path integral and extract the matrix elements from appropriate ratio of correlation functions [19].

The result of our analytic calculation of the pion form factor at moderate and 
large momentum transfer [18 is reported in Fig 2 The instanton contribution is $o(\kappa)$, i.e. maximally enhanced. The ILM can quantitatively reproduce the available data and explain the deviation from the perturbative regime at large momentum transfer.

Conversely, we have observed that in the $\gamma \gamma^{*} \rightarrow \pi_{0}$ transition form factor the instanton effects are of order $o\left(\kappa^{2}\right)$, hence parametrically suppressed by an additional power of the packing fraction. This explains the early onset of the perturbative regime in such a form factor. Moreover, a calculation of the pion light-cone distribution amplitude in the ILM was performed in [21. It was found that instantons can explain the behavior of the low-energy experimental data $\left(Q^{2}<2 \mathrm{GeV}^{2}\right)$ for the $\gamma \gamma^{\star} \rightarrow \pi^{0}$ transition form factor.

Our ILM calculation of the proton electric form factor $G_{E}\left(Q^{2}\right)$ is reported in Fig 2 (right panel), where it is compared with a SLAC data at low $Q^{2}$ and of the JLAB data at large- $Q^{2}$. Again, the short-range correlations induced by instantons quantitatively explain the experimental data. Similar results have been obtained also for the magnetic form factor [19].

Physically, the fact that instantons give very hard hadronic form factors can be interpreted as follows. Due to the strong zero-mode attraction, the hadron wavefunction in coordinate space is very narrow and peaked around the origin. As a consequence, the charge distribution changes very rapidly at short distances (see for example Fig. 31 right panel) leading to large hard-momentum components in its Fourier transform (i.e. the form factor). However, due to the finite size of the instanton, the zero-mode attraction cannot transfer infinitely large momenta. Hence, the electric charge distribution has to become eventually flat, for distances much smaller than the instanton size. Indeed, when $Q^{2} \gtrsim 10-15 \mathrm{GeV}^{2}$, we have observed that the zero-mode contribution to the electro-magnetic correlation function rapidly dies-out and the perturbative regime is finally free to set-in. In conclusion, the ILM predicts that the perturbative limit will not be reached, in the kinematic region accessible to the forthcoming JLAB experiments.

Instantons also provide a possible explanation of why DIS leading-twist perturbative evolution equations works so well, already for $Q^{2} \gtrsim 1 \mathrm{GeV}^{2}$. Lee, Weiss and Goeke 22] have shown that the instanton-contribution to the twist 3 operators in the Operator Product Expansion is parametrically suppressed by powers of the packing fraction $\kappa$, in complete analogy with what we found in the $\gamma \gamma^{*} \rightarrow \pi_{0}$ transition form factor. Moreover, Ostrovsky and Shuryak have recently shown that the ILM can explain the available data on azimuthal spin asymmetries in DIS [23.

\section{Instantons and the $\Delta I=1 / 2$ rule for hyperon decays}

As we have already mentioned, non-leptonic weak decays of hyperons are good testing grounds for spin-dependent, non-perturbative instanton correlations. The decay amplitudes can be parametrized in terms of two constants, corresponding to 
Table 2. Random Instanton Liquid Model prediction and experimental results for P-wave and S-wave amplitudes for non-leptonic weak decays of hyperons. Following the standard notation, $B_{q}^{Q}$ corresponds to $\operatorname{Amp}\left(B^{Q} \rightarrow B^{\prime}+\pi^{q}\right)$.

\begin{tabular}{ccccc}
\hline $\begin{array}{c}\text { Amplitude } \\
\left(\times 10^{7}\right)\end{array}$ & P-wave (theory) & P-wave (experiment) & S-wave (theory) & S-wave (experiment) \\
\hline$\Lambda_{0}^{0}$ & $-10.9 \pm 1.17$ & $-15.61 \pm 1.4$ & $-1.75 \pm 0.34$ & $-2.36 \pm 0.03$ \\
$\Lambda_{-}^{0}$ & $17.71 \pm 1.66$ & $22.40 \pm 0.54$ & $2.25 \pm 0.57$ & $3.25 \pm 0.02$ \\
$\Sigma_{0}^{+}$ & $22.4 \pm 3.55$ & $26.74 \pm 1.32$ & $-3.55 \pm 0.64$ & $-3.25 \pm 0.02$ \\
$\Sigma_{+}^{+}$ & $31.84 \pm 4.81$ & $41.83 \pm 0.17$ & 0 & $0.14 \pm 0.03$ \\
$\Sigma_{-}^{-}$ & $-1.52 \pm 0.30$ & $-1.44 \pm 0.17$ & $4.34 \pm 0.90$ & $4 . .27 \pm 0.01$ \\
$\Xi_{-}^{-}$ & $14.15 \pm 2.75$ & $17.45 \pm 0.58$ & $-4.22 \pm 0.82$ & $-4.49 \pm 0.02$ \\
$\Xi_{0}^{0}$ & $-10.42 \pm 1.95$ & $-12.13 \pm 0.71$ & $3.20 \pm 0.58$ & $3.43 \pm 0.06$ \\
\hline
\end{tabular}

parity-violating and parity-conserving transitions:

$$
\left\langle B^{\prime} \pi\left|H_{e f f}\right| B\right\rangle=i \bar{u}_{B^{\prime}}\left[A-B \gamma_{5}\right] u_{B} .
$$

$H_{\text {eff }}$ is the effective Hamiltonian, which incorporates the electro-weak and the hard-gluon contributions, $B\left(B^{\prime}\right)$ denotes the initial (final) baryon, and $A$ and $B$ are respectively called $S$-wave and $P$-wave amplitudes, each of which can be decomposed in $\Delta I=1 / 2$ and $\Delta I=3 / 2$ components. The $\Delta I=1 / 2$ transition amplitudes are found to be typically $\sim 20$ times larger that the $\Delta I=3 / 2$ amplitudes ( " $\Delta I=1 / 2$ " rule).

We have calculated these amplitudes in the ILM 24]. Our results are reported and compared with the experiment in table 1 We have found that not only instantons can explain the $\Delta I=1 / 2$ rule, but also that the theoretical predictions for the amplitudes were in quantitative agreement with experiment. Note that a $\sim 20 \%$ discrepancy between theory and experiemt is of the same order of the uncertainty which was introduced by assuming the flavor $S U(3)$ limit in the calculation.

In [25] Kochelev and Vento (KV) computed the instanton contribution to nonleptonic kaon decays. On a qualitative level, they found a similar result: the inclusion of the instanton effects indeed produces a strong enhancement of the $\Delta I=1 / 2$ decay channel. On a quantitative level, such an enhancement was found to be still insufficient to reproduce the experimental data. However, it should be mentioned that non-leptonic kaon decays in the $\Delta I=1 / 2$ channel receive large contribution also from final-state interactions, which have not been included in the KV analysis. Moreover, it is now clear that the KV calculation is undershooting the instanton contribution $^{\mathrm{a}}$.

\footnotetext{
${ }^{a}$ The KV calculation was performed in the single-instanton approximation. In such an approach, one treats explicitly the degrees of freedom of the closest instanton and introduces an additional parameter $m^{*}$, which effectively encodes contributions from all other instantons. In their calculation, the authors adopted the phenomenological estimate for $m^{*}$ which was available at the time, $m^{*} \simeq 260 \mathrm{MeV}$. Later, the same parameter was rigorously defined, and determined from numerical simulations in the ILM [20], It was found to be considerably smaller $\left(m^{*} \simeq 80 \mathrm{MeV}\right)$.
} 


\section{Instantons and diquarks}

The instanton-induced interaction (11) in particularly attractive in the $0^{+}$anti-triplet diquark channel. The question whether such interaction leads to binding has been first posed in [26. In an exploratory study based on numerical calculation of pointto-point correlation functions [28, Shuryak, Schäfer and Verbaarshot found some indication of an instanton-induced deeply bound $0^{+}$anti-triplet diquark, with a mass of roughly $450 \mathrm{MeV}$. On the other hand, Diakonov and collaborators have analyzed the same channel by solving Schwinger-Dyson equations at the meanfield level (leading order in $1 / N_{c}$ ) [29]. They found evidence for correlations, but no binding ${ }^{\mathrm{b}}$. The role of instanton-induced diquark correlations in the nucleon has been also investigated in the context of QCD sum-rules [27].

In order to clarify whether instantons do or do not generate a scalar diquark bound-state, we have computed numerically the diquark correlation function

$$
G 2(\tau)=\int d^{3} \mathbf{x}\left\langle 0\left|T\left[J_{D}(\mathbf{0}, \tau) J_{D}^{\dagger}(\mathbf{x}, 0) P e^{\int d y_{\mu} A_{\mu}(y)}\right]\right| 0\right\rangle,
$$

where $J_{D}(x)$ is the scalar color-antitriplet diquark interpolating operator and the path-ordered exponent has been inserted to assure gauge-invariance. If there is a bound-state diquark, then in the large Euclidean time limit the logarithm of the two-point function must scale linearly with $\tau$ :

$$
\ln G 2(\tau) \stackrel{\tau \rightarrow \infty}{\rightarrow} \alpha-\tau M_{D},
$$

where $\alpha$ is a constant and $M_{D}$ should be smaller than twice the constituent quark mass. The result of our calculation (which accounts for the instanton-induced interaction to all orders and does not involve the large $N_{c}$ limit) is presented in Fig. 3 (left panel). Clearly, we found unambiguous evidence for a diquark boundstate with $M_{D} \simeq 450 \mathrm{MeV}$ 30.

An important related problem is what is the size of such a diquark. To answer this question we have extracted the diquark electric charge radius from its electromagnetic form factor [30] (see Fig. 3 right panel). We found that $r_{E} \sim 0.7 \mathrm{fm}$ which implies that the size of the diquark is comparable with that of the proton.

\section{Conclusions}

We have reported on the results of a series of investigations on instanton-induced correlations. We have discussed a collection of lattice results which provide indications for large instanton-induced contributions to the dynamics of light quarks. We have shown that the ILM provides a qualitative and quantitative understanding on the global electro-weak structure of the pion and the proton. In particular, it can explain the fact that the short-range non-perturbative correlations are very strong in some reactions and almost absent in some others.

${ }^{\mathrm{b}}$ Except for $N_{c}=2$, in which case the diquark is a baryon and its mass is protected by Pauli-Gursey symmetry. 

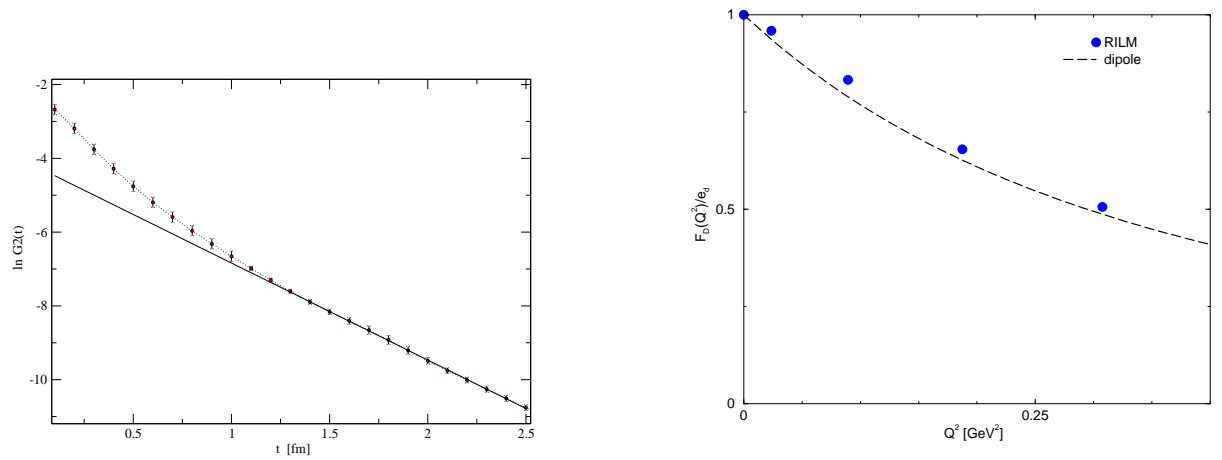

Fig. 3.

LEFT PANEL: Logarithm of the diquark two-point function, $\ln G 2(\tau)$ computed in the Random Instanton Liquid Model. The linear slope is a clean signature of the existence of a bound state. RIGHT PANEL: The diquark form factor (normalized to the total diquark charge) in the Random Instanton Liquid Model (points) compared with the dipole fit parametrizing low-energy data on the proton electric form factor, $F_{d i p}=1\left(1+Q^{2} / 0.71\right)^{2}$ (dashed line).

We have presented a calculation which clearly shows that instantons lead to the formation of a deeply-bound $\left(0^{+}, \overline{\mathbf{3}}_{\mathbf{c}}\right)$ diquark, with a mass of about $450 \mathrm{MeV}$ and size of the same order of that of the proton. The quantum numbers and the mass of the instanton-induced diquark make it a candidate for the "good diquark", claimed by Jaffe and Wilczek [3].

\section{References}

1. J. Volmer et al. [The Jefferson Laboratory $F_{\pi}$ Collaboration], Phys. Rev. Lett. 86 (2001) 1713.

2. M.K. Jones, et al., Phys. Rev. Lett. 84 (2000) 1398. O. Gayou, et al., Phys. Rev. Lett. 88 (2002) 092301.

3. R.L. Jaffe, hep-ph/0409065

4. B. Stech, Phys. Rev. D 36 (1987) 975. B. Stech and Q.P. Xu, Z. Phys. C 49 (1991) 491. H.D. Dosch, M. Jamin and B. Stech, Z. Phys. C 42 (1989) 167. M. Neubert, Z. Phys. C 50 (1991) 243. M. Neubert and B. Stech, Phys. Rev. D 44 (1991) 775.

5. G.'t Hooft, Phys. Rev. Lett., 37 (1976) 8. G.'t Hooft, Phys. Rev., D14 (1976) 3432. G. 't Hooft, Phys. Rept. 142, 357 (1986).

6. E.V. Shuryak, Nucl. Phys. B214 (1982) 237.

7. D.J. Dyakonov and V.Yu. Petrov, Nucl. Phys., B272 (1986) 457.

8. T. Schäfer and E.V. Shuryak, Rev. Mod. Phys. 70 (1998) 323.

9. T. DeGrand and A. Hasenfratz, Phys. Rev. D 64, 034512 (2001). T. DeGrand and A. Hasenfratz, Phys. Rev. D 65, 014503 (2002). I. Hip, T. Lippert, H. Neff, K. Schilling and W. Schroers, Phys. Rev. D 65, 014506 (2002). R. G. Edwards and U. M. Heller, Phys. Rev. D 65, 014505 (2002). T. Blum et al., Phys. Rev. D 65, 014504 (2002). C. Gattringer, M. Gockeler, P. E. Rakow, S. Schaefer and A. Schäfer, Nucl. Phys. B 617, 101 (2001). S. J. Dong et al., Nucl. Phys. Proc. Suppl. 106, 563 (2002).

10. P. Faccioli and T.A. DeGrand, Phys. Rev. Lett. 91 (2003) 182001.

11. P. Faccioli, hep-ph/0211383 
12. T. DeGrand, Phys. Rev. D 64 (2001) 094508.

13. T. Schafer and E. V. Shuryak, Phys. Rev. Lett. 86(2001) 3973.

14. R. L. Jaffe and F. Wilczek, Phys. Rev. Lett. 91(2003) 232003.

15. P. Faccioli and E. V. Shuryak, Phys. Rev. D 65 (2002) 076002.

16. P. Faccioli, A. Schwenk and E. V. Shuryak, Phys. Lett. B 549 (2002) 93.

17. P. Faccioli, Phys. Rev. D 65(2002) 094014.

18. P. Faccioli, A. Schwenk and E.V. Shuryak, Phys. Rev. D67 (2003) 113009.

19. P. Faccioli, Phys. Rev. C 69 (2004) 065211.

20. P. Faccioli and E.V. Shuryak, Phys. Rev. D64 (2001) 114020.

21. A. E. Dorokhov, JETP Lett. 77 (2003) 63 [Pisma Zh. Eksp. Teor. Fiz. 77, 68 (2003)].

22. N. Y. Lee, K. Goeke and C. Weiss, Phys. Rev. D 65 (2002) 054008.

23. D. Ostrovsky and E. Shuryak, hep-ph/0409253

24. M. Cristoforetti, P. Faccioli, E. V. Shuryak and M. Traini, Phys. Rev. D70(2004) 054016.

25. N.I. Kochelev and V. Vento, Phys. Rev .Lett. 87 (2001) 111601.

26. D. Diakonov and V. Petrov, "Diquarks in the instanton picture", in the proceedings of the conference "Quark Cluster Dynamics", Bad Honnef, 29 June - 1 July 1992, Springer.

27. A.E. Dorokhov and N.I. Kochelev, Z. Phys. C46 (1990), 281.

28. T. Schaefer, E.V. Shuryak and J. Verbaarschot, Nucl. Phys. B412 (1994) 143.

29. D. Diakonov, H. Forkel and M.Lutz, Phys. Lett. B373 (1996) 147. D. Diakonov and G.W. Carter, Phys. Rev. D60 (1999) 016004.

30. M. Cristoforetti, P. Faccioli, G. Ripka and M. Traini, hep-ph/0410304 